\title{
Defining Health Profession Regulators' Roles in the Canadian Healthcare System
}

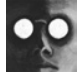 \\ INTRODUCTION \\ Joshua Tepper*, MD, MPH, MBA, FCFPC \\ President and CEO \\ Health Quality Ontario \\ Toronto, ON \\ Humayun Abmed, BA \\ Institute of Health Policy, Management and Evaluation \\ University of Toronto \\ Toronto, ON \\ Adalsteinn D. Brown, DPнгц \\ Editor-in-Chief, Healthcare Papers \\ Director, Institute of Health Policy, Management and Evaluation \\ Dalla Lana Chair in Public Health Policy \\ Dalla Lana School of Public Health, University of Toronto \\ Toronto, ON
}

Health Professions Regulation today faces a myriad of challenges, due to both the perceived performance of regulatory colleges, how health systems have evolved, and even larger political and economic shifts such as the renegotiation of NAFTA. In this issue of
Healthcare Papers, Wilkie and Tzountzouris (2017) describe the work of the College of Medical Laboratory Technologists of Ontario (CMLTO) to redefine professionalism in the context of these challenges. Their paper, and the comments of the responding authors in 
this issue highlight that there, is an overarching perception that health regulatory structures across a range of professions - are not working as effectively as they should. Across this issue of Healthcare Papers, attention is drawn to the fact that more can be done to improve both the function and perception of professional regulatory bodies. However, each paper presents a different approach to how improvements in function and perception are possible.

The first issue is that there is substantial variation in the approach of regulators across professions and across provinces. Kendel (2017) provides rich personal reflections on the need to build effective interprofessional collaboration (IPC) by building trust and appreciation among team members. This can be reflected by regulatory bodies recognizing and acknowledging overlapping spheres of competence, and not giving in to pressure exerted by members of their own college to defend or expand their scope as their primary objective. Saks (2017) encourages regulators to develop a greater understanding of the theoretical and philosophical complexities encompassing regulation and to take cues from analogous regulatory systems (e.g., the UK's) in advocating for changes to legislation and policy. Leslie and Nelson (2017) point out the urgent need for colleges to declare clearly and publicly their dedication to protecting public interests through transparency and engagement and to proactively pursue reforms to restore the level of public confidence necessary for self-regulation.

Penney and Wainwright (2017) emphasize the need for regulatory bodies to respond to their current context by jointly developing healthcare problem definitions with other stakeholders and responding to them through initiatives that engage interdisciplinary models. Wenghofer and Kam (2017) provide a perspective on the somewhat separate evolution of professions and their colleges by pointing

towards shared regulatory responsibilities and the need for the colleges to "un-silo" (i.e., unsegment and unisolate) their regulatory practices. Austin (2017) reflects on the work done by Wilkie and Tzountzouris in acknowledging the competing pressures faced by any regulatory body, including public protection, members' accountability, and members' expectations. Oetter and Johansen (2017) emphasize the role of best organizational practices, and the need to clearly situate regulatory actions as in the public interest to reduce public concerns about professional advocacy and conflict of interest.

Together, the lead paper and commentaries outline a number of approaches to meet the challenges facing regulators today. Most of the papers, explicitly or implicitly, note these evolving challenges are matched by the similarly evolving and sometimes poorly understood role of health professions regulators.

... there have been important increases in what different providers can clinically do...

\section{Current Status}

Many of the regulatory structures that are in place today are the result of policy pressures and health system dynamics from previous decades, when both the delivery of healthcare and public expectations of the healthcare system were different. There has been a steady increase in the expectation of transparency across the entire public sector. At the same time, there have been important increases in what different providers can clinically do based on new knowledge, technology and medications. This has been matched by a substantial evolution in what the public expects of providers that extends beyond clinical care to include the concept of professional 
behaviour. This later change has encouraged health regulatory colleges to extend their oversight well beyond competence (i.e., clinical knowledge and skills) and traditional notions of professionalism to include engagement with families, patients, and other caregivers - as discussed in the lead paper (Wilkie and Tzountzouris 2017) - as well as to questions around how their business practices are organized. Once again this evolution has progressed at different rates and over different periods of time across colleges.

The understanding of "quality care" has broadened to include: safety, effectiveness, patient experience, equity, efficiency, and timeliness (IOM 2001). The way we measure quality across health systems has also changed at the same time from the traditional approach focused on competence and appropriateness to one that reflects these broader domains of quality. There has also been a parallel shift away from a traditional reactive (e.g., complaintsdriven) approach to a more proactive (e.g., periodic inspection) approach. The performance measurement and quality improvement revolutions in healthcare along with more broad movements towards new public management approaches that emphasize performance measurement and management, present a much different and more transparent approach to health system oversight. As virtually all of the papers note, healthcare professionals are no longer isolated, but instead work in teams, relying on often overlapping competencies. Interprofessional collaboration is a reality of the modern healthcare system and its oversight should be based in regulatory structures that no longer work in individual "bunkers," but instead appreciate and include other groups in their shared aim of quality. Finally, several regulators also function in an environment where substantial concerns exist about the rising burnout, depression, suicide and addiction rates of their members and where the high level of physical and verbal violence against their members is increasingly documented.

While these changes are happening, regulators around the world and their professions have faced significant public scrutiny in their handling of certain issues including: sexual abuse of patients by providers, the public release of an unauthorized colonoscopy recording, and contentious Facebook interchanges between providers. These types of incidents have increased the number of issues to which colleges may feel they have to respond. This has led to higher levels of public engagement, scrutiny, and transparency by health professions regulators. And without other information that would be typical of new public management approaches that could describe system functioning overall, colleges are vulnerable to critiques that they exist to protect guild-like professional structures or a countervailing view held by some professionals that the regulatory bodies exist to unfairly persecute members.

... colleges are vulnerable to critiques that they exist to protect guild-like professional structures...

\section{Moving Forward}

Given these changes in our healthcare system and challenges to our regulatory bodies - what should be the role and expectations for regulators? The papers in this issue suggest three pillars to any regulator's mission, all of which are based on the overriding goal of protecting the public. The first role is to provide oversight of a profession by enforcing entry-to-practice standards, selecting out practitioners who fail evaluations of entry-level competence and professionalism. The second role is to ensure sustained, life-long competence and professionalism through activities that analyze and elevate the competence, professionalism, and quality of healthcare delivery of all the members of the college through 
reactive and proactive mechanisms. Finally, the role of the regulatory body is to act as an advocate of quality within the profession as a whole as part of its broader goal of public protection.

In achieving these three roles healthcare professions can still make a reasoned and strong argument for the importance of some type of self-regulation. Unfortunately there is no clearly dominant or preferable approach to reforming self-regulation. However, professions and their colleges will likely need to modify their approach with generally greater degrees of lay governance, ensuring strong and transparent governance and empowering more strongly a public and patient voice to help ensure both high quality practice and engaged providers. This means that colleges will need to turn to each other to look for best practices that can be assembled into reform programs that fit each profession. To ensure that efforts towards better performance are sustainable and touch on all college roles, it is necessary to isolate all existing gaps in regulatory college performance; this, in turn, begins with evaluating and measuring the existing performance and effectiveness of regulatory colleges. Such analysis will help clarify both the "what" and the "how" of the journey the health professions regulator should undertake in order to eventually achieve optimal performance and oversight.

\section{References}

Austin, Z. 2017. "Enhancing the Relationship Between Regulators and Their Profession." Healthcare Papers 16(4): 48-52. doi:10.12927/hcpap.2017.25200.

Institute of Medicine (IOM). 2001. Crossing the Quality Chasm: A New Health System for the 21 $1^{\text {st }}$ Century. Washington, DC: National Academy Press.

Kendel, D. 2017. "Medical Laboratory Technologists as Quality Improvement Team Members." Healthcare Papers 16(4): 34-37. doi:10.12927/hcpap.2017.25203.

Leslie, K. and S. Nelson. 2017. "Ensuring Proactive Regulatory Initiatives Align with the Public Interest." Healthcare Papers 16(4): 42-47. doi:10.12927/ hсpap.2017.25201.

Oetter, H.M. and C. Johansen. 2017. "Acting in the Public Interest: The Heart of Professional Regulation." Healthcare Papers 16(4): 53-57. doi:10.12927/hcpap.2017.25199.

Penney, C. and A. Wainwright. 2017. "Using Trends to Inform Regulatory Practices." Healthcare Papers 16(4): 38-41. doi:10.12927/hcpap.2017.25202.

Saks, M. 2017. "Professional Healthcare Regulation and Practice: The Case of Medicine in Britain." Healthcare Papers 16(4): 23-27. doi:10.12927/ hcpap.2017.25205.

Wenghofer, E.F. and S.M. Kam. 2017. "Evolving Professional Regulation: Keeping up with Health System Evolution." Healthcare Papers 16(4): 28-33. doi:10.12927/hcpap.2017.25204.

Wilkie, K. and J. Tzountzouris. 2017. "Enabling Evolving Practice for Healthcare Professionals: A Regulator's Journey." Healthcare Papers 16(4): 8-21. doi:10.12927/hcpap.2017.25206.

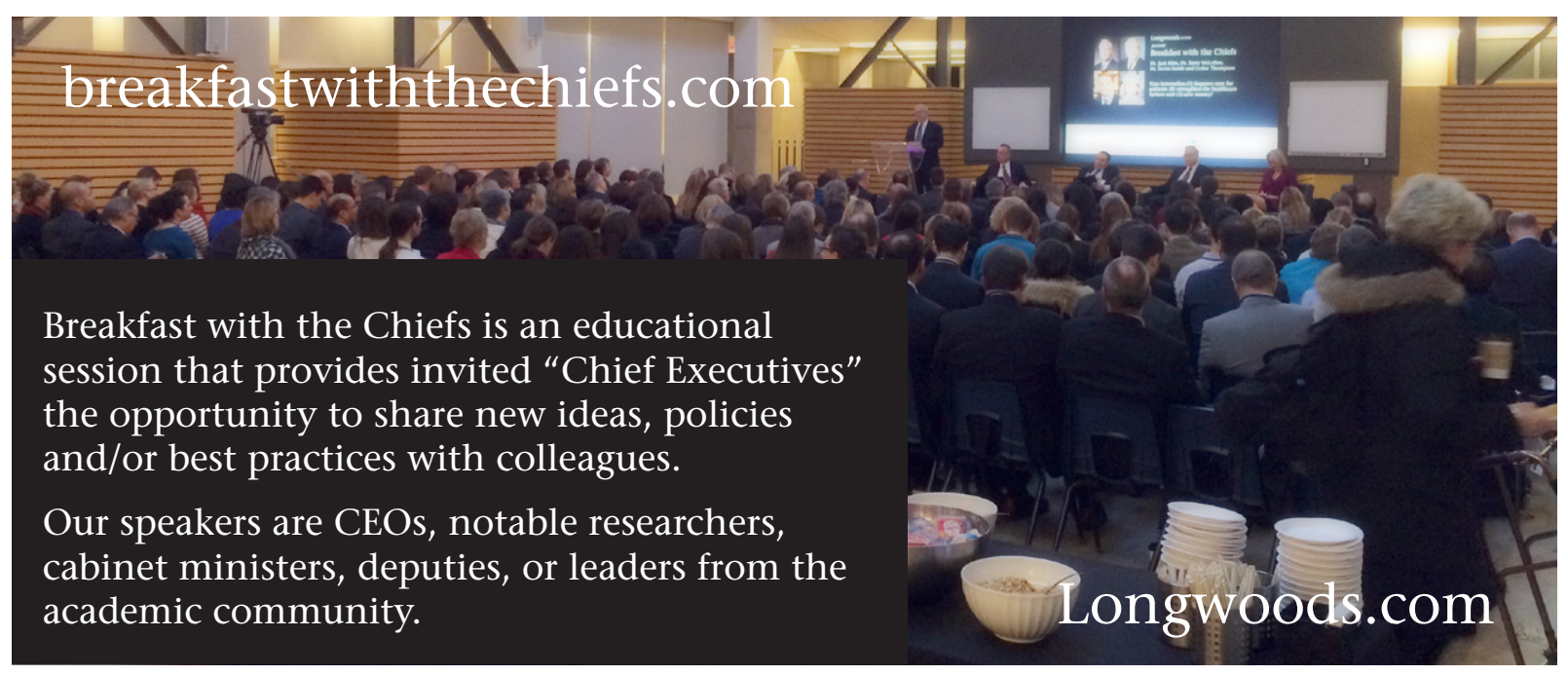

